\author{
...how can the editorial process safeguard \\ people from hasty and inappropriate \\ publication?
}

\title{
OPINION
}

\section{Finding the balance}

Recently the Frost on Sunday television programme in the UK raised a point that dramatically illustrated a constant dilemma for editors of scientific journals, like myself. The point was made (quite emphatically) by Edwina Currie during her review of the Sunday newspapers that the editor of the Lancet should take some of the responsibility and blame for the deaths and illnesses of children who contracted measles because their parents were dissuaded from having their children vaccinated with the MMR vaccine. The reason for the editor's public chastisement was that the Lancet had published the original research paper suggesting there may be a link between the MMR and autism. As we all know this was picked up by the media and created public concern ever since.

The other presenter on the show, Professor Lord Robert Winston, while being careful not to agree on blame and responsibility of the individual editor, agreed that there was a problem in reporting science to the public. He said that he thought that scientists and government had not yet managed to communicate the findings and relevance of science to the public in an effective manner, as was obvious by the current difficulties in the acceptance and development of GMR crops, which as Lord Winston pointed out, had a potentially significant role to play in helping reduce the massive problems in countries where much of the population suffers from starvation.

This difficulty in communication between scientists and readership may not be as stark in dentistry, but the potential for public confusion and resultant damage to patients still exists. Papers published in the $B D J$ are often promoted to the media (or in some cases selected by the media as well) and I need to take this into account when I am making decisions on acceptance or rejection of manuscripts.

Thus the final decision as to which papers we publish depends on much more than simply the accuracy and legitimacy of the research, the opinions of the authors or the importance and relevance of the topic. I must also take into account the perception of the paper by both the general readership (mainly dental practitioners) and the public, usually via the media. Unfortunately the 'spin' that the media can put on the findings of a paper is not only outside our control, but also often open to misintepretation. We see this most often in the reporting on topics such as amalgam, fluoride and cross infection, where the messages communicated to the public via television programmes and newspapers can often be inaccurate and cause unnecessary concern.

With this in mind I have to determine how we strike the right balance between alerting people over a genuine concern (such as thalidomide) and not causing unnecessary harm through inappropriate publication (such as the MMR story). This dilemma especially arises every time a manuscript is submitted which pushes at the boundaries of known and accepted current thinking. While the authors of such a manuscript are convinced they are right - how can the editorial process safeguard people from hasty and inappropriate publication?

While I obviously accept that the $B D J$ needs to stimulate discussion and encourage innovation, I must also consider the perception that publication of such a paper may cause. Regrettably people often confuse publication of a suggested theory or concept as fact, and assume that publication means the idea or theory is true, not just an alternative theory. For this reason this type of paper receives stringent peer review, and is only published if the advisors and referees agree that the ideas in the paper are based on sound scientific thinking and worth publicising to advance thinking and science in the area.

The key word is balance. On one hand we could avoid all controversial and original papers, staying safe. The problem is that this would lead to a stunted and ultimately boring journal - not an option. On the other hand we could 'publish and be damned'. The balance lies somewhere between continuing to encourage new ideas and theories while avoiding confusion and potential harm. The way to achieve that must remain in the peer review process which is the best system we have for trying to ensure we do not stray too far in either direction - either too safe or too dangerous. More of that in my leader next issue.

Mike Grace, Editor m.grace@bda.org

doi: 10.1038/sj.bdj.4811223 